\title{
Research on the influence of design parameters on mechanical performance of net arch bridge
}

\author{
Xinke $\mathrm{Cao}^{1,{ }^{*}, Z h e y u a n} \mathrm{Lu}^{2,{ }^{* *}}, \mathrm{Xianwu} \mathrm{Hao}^{3,{ }^{* * *}, \text { Guojun Yang }}{ }^{4, * * *}$ \\ ${ }^{1}$ University of Chang'an, Department of highway Nanerhuan Road, Xi'an, China, 710064; PH(+86):19829395090; \\ ${ }^{2}$ University of Chang'an, Department of highway Nanerhuan Road, Xi'an, China, 710064; PH(+86):15902985876; \\ ${ }^{3}$ University of Chang'an, Department of highway Nanerhuan Road, Xi'an, China, 710064; PH(+86):13609185024; \\ ${ }^{4}$ Lanzhou university of Technology, 287 langongping Road, Lanzhou, China, 730050; PH(+86):18794209992;
}

\begin{abstract}
In order to study the suspender layout parameters and design parameters of the tied arch bridge with mesh suspenders under the action of vehicle load, the structure stress is more reasonable and meets the higher economy and aesthetics. Taking a $96 \mathrm{~m}$ span reticulated tied arch bridge as the engineering background, the finite element model is established by using Midas/Civil 2019 program. The variation law of internal force and Suspender Force of the structure is calculated and analysed under the change of rise span ratio and suspender number parameters, and the relatively optimal value range of corresponding parameters is given. The results show that the rise span ratio should be 0.2-0.24; The number of Suspenders for one side arch rib should be 34-38; The relatively optimal range of the above parameters is discussed for reference.
\end{abstract}

\section{Introduction}

Tied arch bridge is a combined system composed of arch rib, suspender, tie beam and bridge deck system. It is characterized by external statics, internal statics and tie beam bearing horizontal thrust of arch foot. It can adapt to different geological conditions and is a long-span bridge form with good development prospects. Compared with other types of bridge, tied arch bridge is disadvantageous by bridge circles at home and abroad because it makes full use of the structural performance of arch rib compression and tied beam bending, and has the advantages of various bridge types, economic beauty, large span capacity, small building height and various construction methods ${ }^{1}$.

In recent years, the development of concrete-filled steel tubular arch bridge in China is rapid, especially in highway bridge, but most of them are vertical suspender layout ${ }^{4}$. At present, there are some achievements in the study of mechanical behavior of tied arch bridge ${ }^{5}$. Xiong Hongxia and Wang $\mathrm{Yi}^{7}$ studied the influence of arch rib steel ratio, arch axis coefficient and other parameters on the mechanical behavior of CFST Tied Arch Bridge; Dong-Sheng et al. ${ }^{5}$ studied the parameter analysis of the bearing capacity of tied arch bridge; Sun Jianping et al. ${ }^{10}$ studied the relationship between the stability and parameters of CFST Tied Arch Bridge with transverse brace, arch rib inclination angle and suspender characteristics as parameters. However, there are many researches on the flexible vertical suspender of concretefilled steel tube under various loads in the existing literature, while there are relatively few researches on the rigid mesh suspender under highway loads. Therefore, combined with the actual engineering background, the author will select the rise span ratio, the number of suspenders, the inclination angle of suspenders and the bending stiffness ratio of arch beam and other parameters of tied arch bridge to study the influence of highway load on the structure, which will provide important reference for the future design of tied arch bridge with more economic and better mechanical performance.

\section{Project overview}

Taking a reticulated tied arch bridge as the research object, the calculated span is $96 \mathrm{~m}$, the rise span ratio is $1 / 5$, and the rise height of the main span is $19.2 \mathrm{~m}$. The two arch rings are of concrete-filled steel tube structure and arranged in parallel. The spacing between arch ribs is $13.45 \mathrm{~m}$. The arch axis is a quadratic parabola. Five cross braces and two k-braces are set between the two arch ribs; The cross section of arch rib adopts dumbbell section, the steel pipe material is Q235, the outer diameter of steel pipe is $1 \mathrm{~m}$, the wall thickness is $0.014 \mathrm{~m}$, the wall thickness of batten plate between two steel pipes is $0.014 \mathrm{~m}$, and the batten plate spacing is $0.65 \mathrm{~m}$. The suspenders are symmetrically arranged, and PES (FD) 7-61 low stress anti-corrosion cables are used with a spacing of $5 \mathrm{~m}$. The tie beam is a prestressed concrete box girder structure with a height of $2 \mathrm{~m}$ and a width of $1.2 \mathrm{~m}$; The bridge deck is $14.8 \mathrm{~m}$ wide, belonging to rigid tied rigid arch. The main section parameters are shown in Table 1 
Table1. Main section parameters of the structure

\begin{tabular}{ccccccc}
\hline Type & Arch Rib & Tie beam & $\begin{array}{c}\text { End } \\
\text { crossbeam }\end{array}$ & $\begin{array}{c}\text { Middle } \\
\text { crossbeam }\end{array}$ & Cross brace & Suspender \\
\hline $\mathrm{A}\left(\mathrm{m}^{2}\right)$ & 0.3534 & 1.64 & 3.055 & 0.89 & 0.0395 & 0.054 \\
$\mathrm{E}\left(\times 10^{8} \mathrm{kN} / \mathrm{m}^{2}\right)$ & 2.06 & 0.345 & 0.345 & 0.345 & 2.06 & 1.95 \\
$\mathrm{I}_{1}\left(\mathrm{~m}^{4}\right)$ & 0.0559 & 0.6476 & 1.2430 & 0.0830 & 0.0830 & \\
$\mathrm{I}_{2}\left(\mathrm{~m}^{4)}\right.$ & 0.1917 & 0.6951 & 1.0204 & 0.1171 & 0.1171 & \\
$\mathrm{I}_{3}\left(\mathrm{~m}^{4}\right)$ & 0.0287 & 0.2673 & 0.6009 & 0.0406 & 0.0406 & \\
\hline
\end{tabular}

\section{Calculation model and parameter selection}

\subsection{Computational model}

When Midas/Civil 2019 is adopted for modeling, slab element is adopted for concrete bridge deck, truss element is adopted for suspender, and beam element is adopted for the rest. The boundary conditions are given according to actual constraints, and the calculation model is shown in Figure 1. In this paper, the model is used to analyse the influence of rise span ratio, suspender number, suspender inclination angle and arch beam stiffness ratio on the force of the mesh suspender tied arch bridge. At the same time, considering the structural treatment, economy and aesthetics of the structure, a relatively optimal parameter range is obtained. When a parameter is analysed, other parameters remain unchanged.

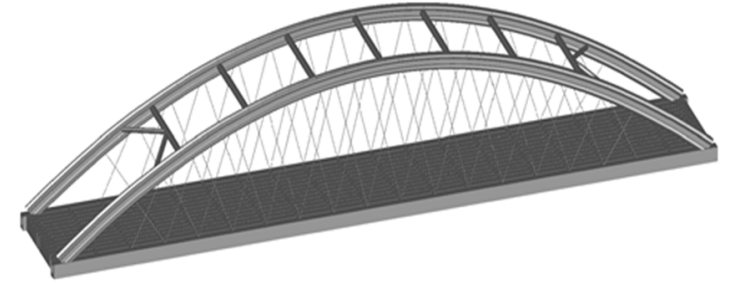

Figure 1. Finite elemgent calculation model

\subsection{Parameter selection}

For arch bridges, bending moment is often one of the most disadvantageous factors; For beams, too large bending moment will lead to the increase of cost; But the fatigue problem of suspender is mainly determined by the magnitude and amplitude of axial force. In view of this, in order to better study the mechanical performance of the mesh suspender, the following mechanical evaluation parameters are selected:

$N_{a}$ : Axial force of arch foot; $q u a N_{a}: l / 4$ Axial force of arch rib; $\operatorname{mid} N_{a}$ : Axial force of vault; $\max N_{a}$ : The maximum axial force of arch rib; $\max M_{a}$ : Maximum bending moment of arch rib;

$N_{\mathrm{g}}$ : Axial force at beam end; quaN $N_{\mathrm{g}}: l / 4$ Axial force of tie beam; $\operatorname{mid} N_{\mathrm{g}}$ : Axial force of tie beam midspan; $\max N_{\mathrm{g}}$ : Maximum axial force of tie beam; $\max _{g}$ : The maximum bending moment of tie beam; $\max N$ : The maximum value of suspender axial force; aveN : Average axial force of all hangers.

\section{Parameter analysis and results}

\subsection{Influence of rise span ratio}

Generally speaking, the rise span ratio of CFST Tied Arch Bridge ranges from 0.2 to 0.25 for the network suspender arrangement. Based on the engineering background, the author selects 7 cases with rise span ratio of $0.2,0.4,0.8$, 2, 6, 10 and 14 .

In this analysis, other geometric parameters remain unchanged( $l=96 \mathrm{~m} ; \lambda=0.1074 ; \quad N_{u}=22$; Arch axis alignment: quadratic parabola), only the rise span ratio is changed, and the finite element models are established respectively.

(Note: $l: \operatorname{span} ; \lambda=(\mathrm{EI})$ arch rib $/(\mathrm{EI})$ indicates the bending stiffness of arch beam; $N_{u}$ :Number of Suspenders for one side arch rib; $\theta$ :Angle between suspender axis and arch rib normal; The meaning of the symbols is not repeated.)

Figure 2 and Figure 3 respectively show the curve of the relationship between the internal force of arch rib and tie beam and the ratio of midsagittal span of the arch rib and tie beam of the mesh suspender tied arch bridge under live load. In order to facilitate comparison, the internal force value of the ratio of vector to span is 0.14 as the benchmark for normalization. 


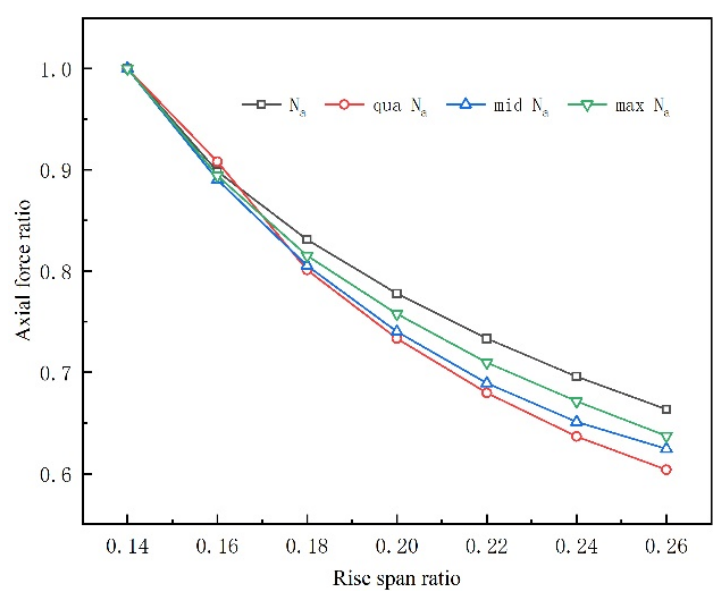

(a) Axial force of arch rib

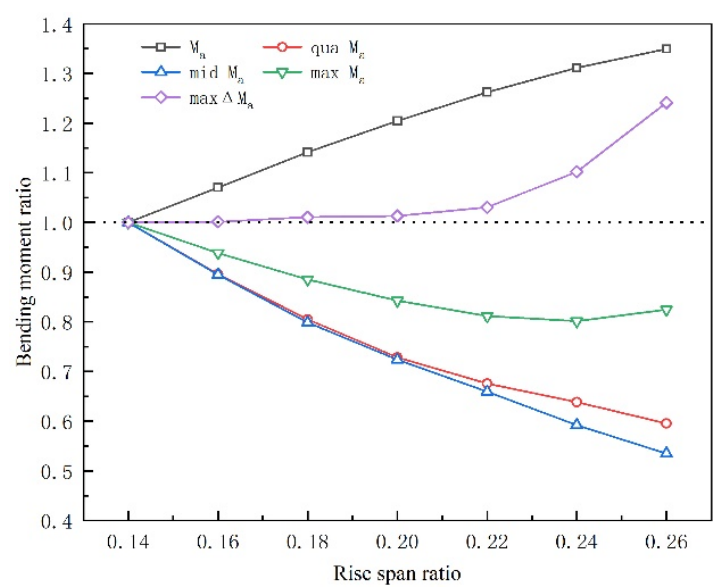

(b) Bending moment of arch rib

Figure 2. Relationship between internal force of arch rib and rise span ratio under vehicle load

It can be seen from Figure $2 \mathrm{a}$ that with the increase of rise span ratio. The axial force level of arch rib shows a decreasing trend, and the descending speed is faster when the rise span ratio is small, but the descending speed is slower when the rise span ratio exceeds 0.2 . When the rise span ratio changes from 0.14 to 0.26 , the axial force of arch foot, arch crown and the maximum axial force of arch rib decrease little, but the axial force of arch rib decreases most significantly, up to $39.6 \%$. It can be seen from Figure $2 \mathrm{~b}$ that with the increase of rise span ratio, the bending

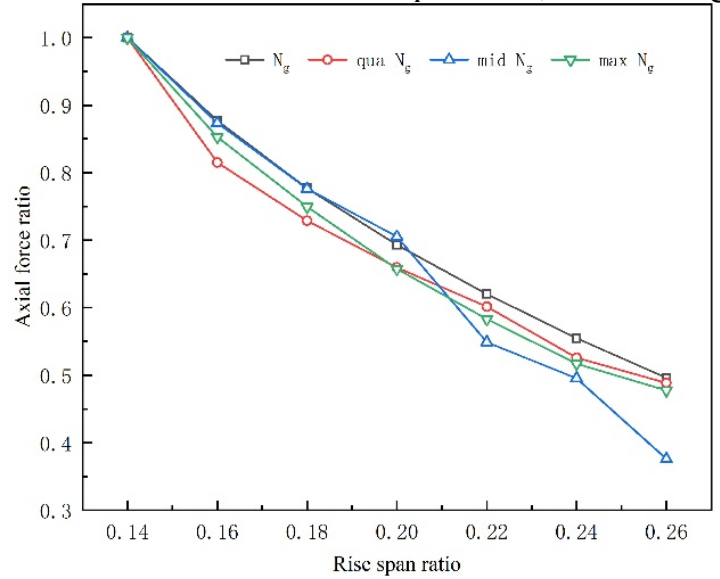

(a) Axial force of tie beam moment of each section of arch rib changes differently, the bending moment of arch rib and vault decreases and the descending curves almost coincide, while the bending moment of arch foot increases. Among them, the most obvious reduction of bending moment on the vault is $46.5 \%$. When the rise span ratio is about 0.22 , the bending moment of the vault reaches the minimum; When the rise span ratio exceeds 0.22 , the vault bending moment and the maximum bending moment increase sharply.

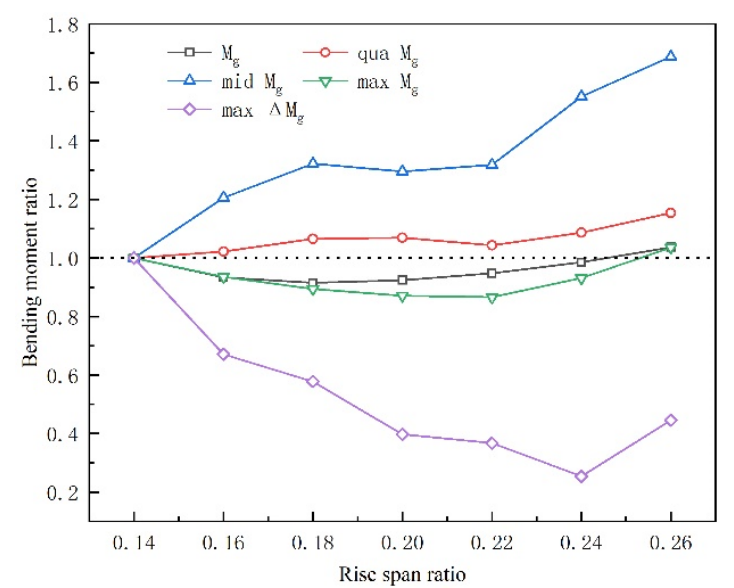

(b) Tie beam bending moment

Figure 3. Relationship between internal force of tie beam and rise span ratio under vehicle load

It can be seen from Figure $3 a$ that with the increase of rise span ratio, the axial force of tie beam presents a downward trend, and the decrease range is more significant. When the rise span ratio increases from 0.14 to 0.2 , there is little difference in the decrease of axial force at all parts of the tie beam. When the rise span ratio exceeds 0.2 , the axial force in the middle of the tie beam decreases rapidly, and the decrease rate is as high as $62.4 \%$. It can be seen from Figure $3 \mathrm{~b}$ that with the increase of rise span ratio, the change trend of bending moment at each part of tie beam is different, and the bending moment at beam end and mid span first decreases and then increases; The maximum moment increment shows a decreasing trend, while the moment of tie beam and tie beam midspan fluctuates, but it shows an increasing trend. When the rise span ratio reaches about 0.22 , the maximum bending moment of tie beam reaches the minimum, and when the rise span ratio exceeds about 0.24 , the bending moment of tie beam increases rapidly.

\subsection{Influence of suspender number}

In order to study the mechanical performance of the mesh suspender with different number of suspenders under vehicle load and give a relatively reasonable number of suspenders, this paper keeps other geometric parameters unchanged $\left(l=96 \mathrm{~m} \quad \lambda=0.1074 f / l=1 / 5 ; \quad \theta=30^{\circ}\right.$; Arch axis alignment: quadratic parabola),only change the number of suspenders, select 20, 26, 32, 38, 44, 50, 56, and establish the finite element model respectively. Some finite element models are shown in Figure 4. 


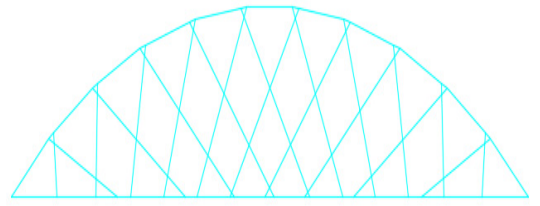

(a) Number of Suspenders for one side arch rib:20

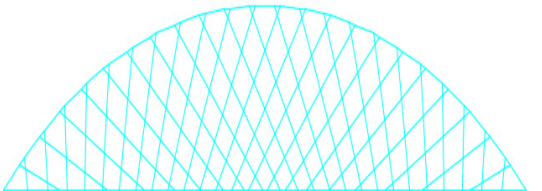

(c)Number of Suspenders for one side arch rib:44 (b) Number of Suspenders for one side arch rib:32

(d) Number of Suspenders for one side arch rib:56

Figure 4. Schematic diagram of calculation model of different suspender number of tied arch bridge with net suspender

Figure 5 shows the curve of maximum bending moment of arch rib and tie beam under the action of vehicle load. Figure 6 shows the change curve of internal force of arch rib and tie beam and Suspender Force under the action of vehicle load and dead load. In order to be more convenient, the internal force value of $N_{u}=38$ is taken as the benchmark to normalize.

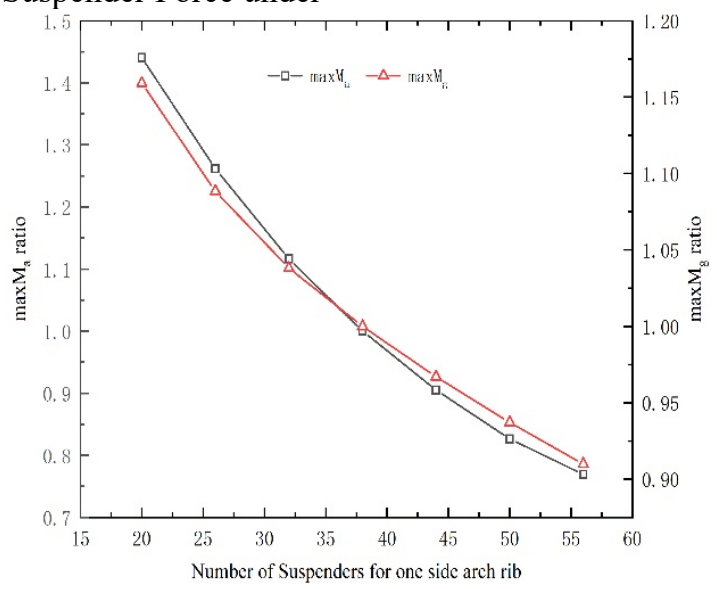

Figure 5. Relationship between structural bending moment and number of suspenders under vehicle load

It can be seen from Figure 6a that with the increase of the number of suspenders, the bending moment of arch rib and tie beam presents a downward trend. When the number of suspenders is about 35 , the maximum bending

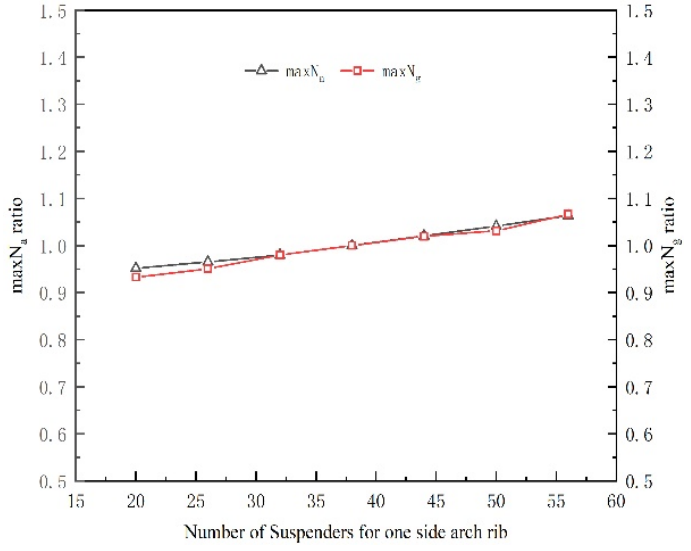

(a) Axial force moment of arch rib and tie beam is equal. When the number of suspenders is more than 35, the maximum bending moment of tie beam decreases slower than that of arch rib.

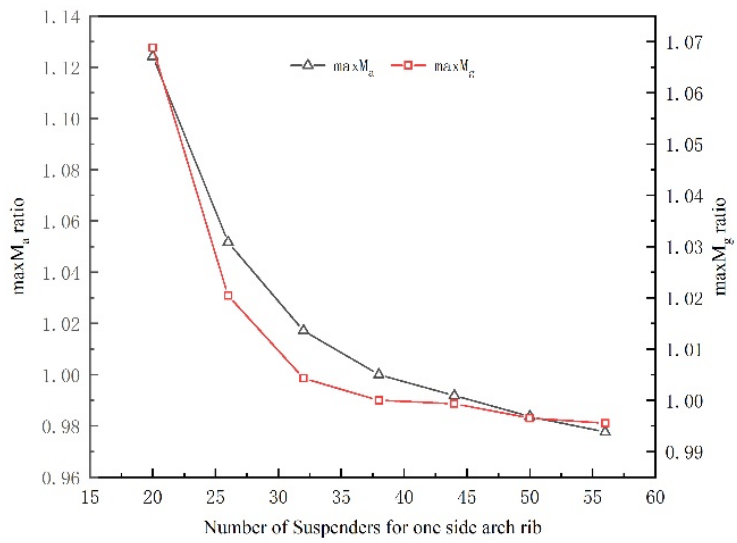

(b) bending moment 


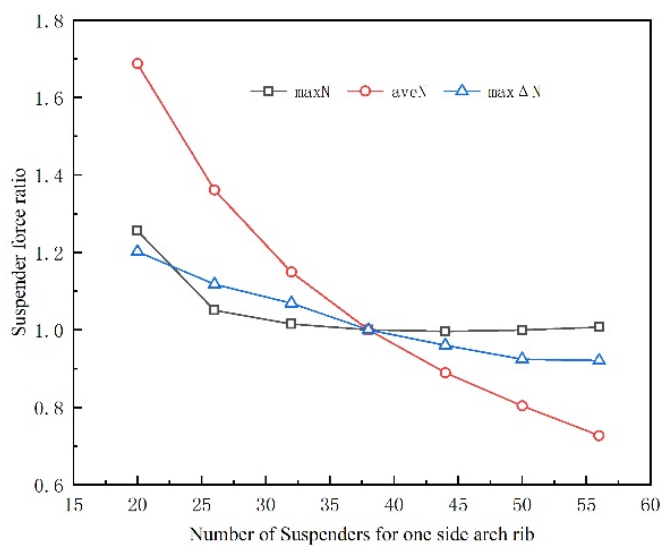

(c) Suspender Force

Figure 6. The relationship between the internal force of structure and the number of suspenders under the action of vehicle load and dead load

Figure 6 a shows that the axial force of tie beam and arch rib is less affected by the number of suspenders on one side arch rib. With the increase of the number of suspenders, the axial force of arch rib and tie beam increases, but the increase is less than $4 \%$. It can be seen from Figure $6 \mathrm{~b}$ that with the increase of the number of suspenders, the bending moment of arch rib and tie beam presents a downward trend, and when the number of suspenders is less than 35 , the maximum bending moment of tie beam decreases faster than that of arch rib; At the same time, it can be seen that when the number of suspenders is about 40 , the maximum bending moment of arch rib and tie beam tends to be relatively stable. When the number of suspenders increases from 20 to 56 , the maximum bending moment of arch rib decreases only by $7.4 \%$, while the maximum bending moment of tie beam decreases by $15 \%$. Obviously, the maximum bending moment of tie beam is more sensitive to the number of suspenders than that of arch rib. It can be seen from Figure $6 \mathrm{c}$ that with the increase of the number of suspenders, the maximum Suspender Force, the average Suspender Force and the variation of the maximum Suspender Force show a decreasing trend. At the same time, it can be seen that when the number of suspenders is 38 , the maximum Suspender Force decreases faster, and the average suspender decreases approximately linearly, but after the number of suspenders is 38 , they tend to be relatively stable.

\section{Conclusion}

In this paper, based on the engineering background, aiming at the equal normal angle suspender layout, the parameters of the mesh suspender tied arch bridge are optimized. The rise span ratio and the number of suspenders are selected, and the corresponding models are established by using the finite element software to study the influence of these structural parameters on the mechanical performance of the structure, The relatively economic and reasonable structure parameter range of medium and small span reticulated suspender tied arch bridge is given:

(1) With the increase of rise span ratio, the axial force of arch rib and tie beam decreases in varying degrees, while the bending moment and the deflection of tie beam first decrease and then increase. Considering the factors of structure stress, construction, economy and aesthetics, the relatively optimal rise span ratio range of medium and small span reticulated suspender tied arch is $0.2 \sim 0.24$ under vehicle load.

(2) With the increase of the number of suspenders in one side arch rib, the bending moment and Suspender Force of arch rib and tie beam decrease in varying degrees, but the axial force of arch rib and tie beam has little effect. Considering the degree of difficulty, economy and aesthetics of construction, it is given that under the action of vehicle load, the number of suspenders of single side arch rib of medium and small span net suspender tied arch is relatively better, ranging from 34 to 38 .

\section{Acknowledgments}

This paper is very grateful for the support of NSFC and China Postdoctoral fund:

Project 1: National Natural Science Foundation of China (Youth Foundation) (51808274)

Project 2: China Postdoctoral Science Foundation (2019m653897xb)

\section{Reference}

1. BAOCHUN C. 2003 J. Journal of Civil Engineering Research Progress on calculation theory of concrete filled steel tubular arch bridge 12 47-57.

2. JUNPING C B L. 2020 J. Journal of Transportation Engineering Summary of arch bridge construction and technology development in the world $020(001)$ 27-41.

3. AL X R E. 2013 Bridge structure system (People's Communications Press)

4. JUNPING C B W J Z J L. 2017 J Journal of Civil Engineering Application status and Prospect of CFST arch bridge in China (6) 50-61.

5. LIU D S. 2007 J Communications Standardization Parameter Study of Ultimate Capacity of Tied-arch Bridges

6. PELLEGRINO C, CUPANI G, MODENA C. $2010 \mathrm{~J}$ Engineering Structures The effect of fatigue on the 
arrangement of hangers in tied arch bridges 32(4) 1140-7.

7. XIONG H, LIU Z, DENG Y. 2008 J Engineering Journal of Wuhan University Analysis of influence of design parameters on mechanical characteristics of concrete-filled steel tubular tied arch bridges

8. MINYUAN H. 2011 J Journal of Central South University of forestry science and technology Reasonable form of CFST rigid frame tied arch bridge 31(002) 90-4.

9. CHUNFENG L, XIUYONG S, ZAISHONG J, et al. 2008 J World Bridge Research on structural system design parameters of steel tied arch bridge 64-7.

10. JIANPING S, RICHEN J, YUEZHEN X. $2011 \mathrm{~J}$ Railway Engineering Study on structural stability design parameters of Railway Long Span tied arch bridge $1226-8$.

11. JIAN W, QINGSONG M. 2011 J Bridge construction Influence of structural parameters on dynamic characteristics of through CFST Arch Bridge

12. ZHENWEI Z, WEI Z. 2018 J Chinese and foreign highways Parameter analysis and linear control of Feiyan CFST Tied Arch Bridge 038(6) 105-8. 